\title{
Lagrangian Strain Tensor Computation with Higher Order Variational Models
}

\author{
Alexander Hewer ${ }^{1}$ \\ hewer@mia.uni-saarland.de \\ Joachim Weickert ${ }^{1}$ \\ weickert@mia.uni-saarland.de \\ Tobias Scheffer ${ }^{2}$ \\ t.scheffer@mx.uni-saarland.de \\ Henning Seibert ${ }^{2}$ \\ h.seibert@mx.uni-saarland.de \\ Stefan Diebels ${ }^{2}$ \\ s.diebels@mx.uni-saarland.de
}

\begin{abstract}
The reliable estimation of the Lagrangian stress tensor from an image sequence is a challenging problem in mechanical engineering. Since this tensor involves first order motion derivatives, it appears tempting to estimate the optical flow field with a highly accurate variational model and compute its derivatives afterwards. In this paper we explain why this idea is inappropriate due to lower order smoothness assumptions and the ill-posedness of differentiation. As a remedy, we propose a variational framework that performs higher order regularisation of the optical flow field and directly computes the Lagrangian stress tensor from the image measurements. Due to its recursive structure, this framework is very generic. It can incorporate smoothness assumptions of arbitrary high order and allows to compute derivatives of any desired order in a stable way. With a biaxial tensile experiment with an elastomer we demonstrate that our novel approach gives substantially better results for the Lagrangian stress tensor than computing derivatives of the optical flow field. Moreover, it also outperforms a frequently used commercial software that marks the state-of-the-art for Lagrangian stress tensor computation.
\end{abstract}

\section{Introduction}

Estimating higher order motion information from an image sequence is a problem that has hardly been addressed in the computer vision community so far. Nevertheless, it can be very important in practical applications. An example is the computation of the Lagrangian strain tensor. This tensor plays a fundamental role in mechanical engineering where it is used to derive properties of materials. Since it involves first order derivatives of the motion field, it extracts second order derivative information from an image sequence. Developing robust methods for this task is challenging. 
Most computer vision research on motion in image sequences focuses on optical flow. Since the optical flow field describes displacements between subsequent frames of an image sequence, it is based on first order derivative information. In the last three decades numerous methods have been developed and an evaluation of the more recent approaches can be found at the Middlebury webpage http://vision.middlebury.edu/flow/. Many of the best performing methods use variational models or related discrete approaches. They obtain the optical flow field as a minimiser of an energy functional that consists of two terms: a data term that models constancy assumptions, and a smoothness term that regularises the flow field and interpolates at those locations where the data term does not provide sufficient information.

Thus, if one is interested in computing the Lagrangian strain tensor, an obvious idea would be to estimate the optical flow field with a modern variational method and to differentiate the result numerically. In general, however, the result will not be satisfying for two reasons: Firstly, most smoothness terms in the optical flow functional penalise large values of first order derivatives of the flow field. This encourages flat or piecewise flat optical flow fields. Hence, the derivatives are biased towards zero and more complex motion information cannot be extracted in a reliable way [ $[0]$. The second problem arises from the numerical differentiation of the result: Since differentiation is a classical ill-posed problem, it would also benefit from regularisation within a variational framework [ $[$ ]].

Our Contribution. The goal of our paper is to address both problems simultaneously: On the one hand, we design a variational model that provides higher order regularisation of the optical flow field. On the other hand, we compute the motion derivatives directly within our energy functional. Thus, there is no need to postprocess the optical flow by numerical differentiation in order to obtain the Lagrangian stress tensor. Last but not least, our framework is very generic: Due to its recursive structure, it is straightforward to include smoothness terms of arbitrary order and to have direct access to motion derivatives of any desired order.

Related Work. Bredies et al. [䧃] and Trobin et al. [四] have found that higher order smoothness assumptions can improve the results of variational approaches. Furthermore,

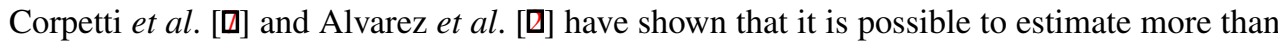
just the motion itself by a variational optical flow method: They also extracted information based on first order motion derivatives. Corpetti et al. restrict themselves to estimating only the divergence and the curl of the motion whereas we can estimate motion derivatives of arbitrary order. Alvarez et al. estimate first order motion derivatives by means of a modified data term. Their method requires to construct and to minimise an energy in every point of the image space. It relies on a initial guess for the motion in order to converge. In our method we can use directly well-established optical flow data terms. Furthermore, we can estimate motion derivatives of arbitrary order, do not need a specific initial guess, and only have to minimise one single energy functional. Both the approaches of Corpetti et al. and Alvarez et al. use second order smoothness assumptions, while our model is designed to allow smoothness terms of arbitrary order.

Organisation of the Paper. In Section 2 we review a baseline variational approach that solves the classical optical flow problem and serves as starting point for our novel framework. 
Furthermore, we discuss why its smoothness term renders this approach unsuitable for deriving higher order motion information such as Lagrangian strain tensor. Then we introduce in Section 3 our generic approach that extends this baseline approach. In particular, we construct methods that can directly estimate motion derivatives up to second order. In Section 4, we give a short overview of our implementation. Section 5 evaluates the performance of our method by applying it to a biaxial tensile experiment that is known for producing complex motions. Our paper is concluded with a summary and an outline of future research in Section 6.

\section{Classical Optical Flow Problem}

Before we can discuss variational methods for computing motion derivatives in image sequences, we first have to understand how a classical variational approach for optical flow estimation works and what are its limitations for our task. This is the goal of the present section.

\subsection{Our Baseline Variational Model}

Assume we are given grey-valued images $f_{k}: \Omega \rightarrow[0,255]$ where $\Omega \subset \mathbb{R}^{2}$ is a rectangular image domain, and the index $k$ describes the frame in the sequence. We want to estimate the displacement field (optical flow) $\mathbf{u}=\left(u_{1}, u_{2}\right)^{\top}: \Omega \rightarrow \mathbb{R}^{2}$ between two consecutive frames $f_{1}$ and $f_{2}$ of this sequence, such that $\mathbf{x}+\mathbf{u}(\mathbf{x})$ is the new position of an object in $f_{2}$ that was originally located at position $\mathbf{x}$ in frame $f_{1}$. In the following, we will often omit the parameters of functions for the sake of brevity. So we will write $\mathbf{u}$ instead of $\mathbf{u}(\mathbf{x})$ if the meaning is clear from the context.

A variational optical flow approach obtains $\mathbf{u}$ as the minimiser of an energy

$$
E(\mathbf{u})=\int_{\Omega}(D(\mathbf{u})+\alpha S(\mathbf{u})) \mathrm{d} \mathbf{x}
$$

where $D$ is a data term and $S$ represents the smoothness term that is weighted by $\alpha>0$.

As we are expecting outliers and illumination changes in the data, we use the data term proposed by Bruhn and Weickert []:

$$
D(\mathbf{u}):=\psi\left(\left|f_{2}(\mathbf{x}+\mathbf{u})-f_{1}(\mathbf{x})\right|^{2}\right)+\psi\left(\left\|\nabla f_{2}(\mathbf{x}+\mathbf{u})-\nabla f_{1}(\mathbf{x})\right\|^{2}\right)
$$

where $\psi\left(s^{2}\right):=\sqrt{s^{2}+\varepsilon^{2}}$ represents the regularised $L_{1}$-norm. The regularisation parameter $\varepsilon$ ensures differentiability in 0 and is set to 0.1 in this work. The first part of this term models the idea that the grey value remains constant over time. The second one represents the assumption that the spatial image gradient stays constant, which makes the approach more robust against illumination changes. The usage of the non-quadratic penaliser $\psi$ provides some robustness against outliers. Additionally, the separate penalisation of both assumptions allows also solutions where only one assumption is fulfilled.

Unfortunately the data may be unreliable in some regions, e.g. due to flat structures, which requires the usage of a smoothness assumption. As we do not expect any discontinuities in the motion, we use the classical smoothness term of Horn and Schunck [四]:

$$
S(\mathbf{u}):=\sum_{i=1}^{2}\left\|\nabla u_{i}\right\|^{2}
$$


This term models the idea that the first order derivatives of $\mathbf{u}$ vanish, which implies that the motion is assumed to be constant in space. In the following, we will refer to terms that represent the idea that the $n$-th order derivatives should vanish as $n$-th order smoothness terms. Furthermore, we will say that $\mathbf{u}$ has $n$-th order smoothness if it results from a variational model with such a term.

\subsection{Euler-Langrange Equations and Boundary Conditions}

In order to find a minimiser of our functional (1), we may solve the associated EulerLagrange equations. These are equations that a minimiser must necessarily fulfill [ $[$ ] . In our case, we obtain two equations:

$$
\begin{aligned}
& 0=\partial_{u_{1}} D(\mathbf{u})-\alpha \Delta u_{1}, \\
& 0=\partial_{u_{2}} D(\mathbf{u})-\alpha \Delta u_{2}
\end{aligned}
$$

where

$$
\begin{aligned}
& \partial_{u_{1}} D(\mathbf{u})=\psi^{\prime}\left(G^{2}\right) \cdot G F_{x_{1}}+\psi^{\prime}\left(G_{x_{1}}^{2}+G_{x_{2}}^{2}\right) \cdot\left(G_{x_{1}} F_{x_{1} x_{1}}+G_{x_{2}} F_{x_{2} x_{1}}\right), \\
& \partial_{u_{2}} D(\mathbf{u})=\psi^{\prime}\left(G^{2}\right) \cdot G F_{x_{2}}+\psi^{\prime}\left(G_{x_{1}}^{2}+G_{x_{2}}^{2}\right) \cdot\left(G_{x_{1}} F_{x_{1} x_{2}}+G_{x_{2}} F_{x_{2} x_{2}}\right)
\end{aligned}
$$

with the abbreviations $F:=f_{2}(\mathbf{u}+\mathbf{x})$ and $G:=f_{2}(\mathbf{x}+\mathbf{u})-f_{1}(\mathbf{x})$.

Moreover, the Euler-Lagrange formalism also involves conditions that specify what happens at the boundaries of $\Omega$. It gives so-called homogeneous Neumann boundary conditions that model a reflecting situation:

$$
\mathbf{n}^{\top} \nabla u_{i}=0 \quad \text { for } i \in\{1,2\}
$$

where $\mathbf{n}$ represents the outer surface normal at the boundary of $\Omega$.

\subsection{Suitability for Computing the Lagrangian Strain Tensor}

Methods for solving the optical flow problem compute motion $\mathbf{u}$ between two images. In some applications, however, information based on motion derivatives is needed as well. The Lagrangian strain tensor is an example for such information. It is defined as [ $\square$ ]

$$
\mathcal{E}=\left(\begin{array}{ll}
e_{11} & e_{12} \\
e_{12} & e_{22}
\end{array}\right):=\frac{1}{2}\left((\nabla \mathbf{u}+\mathbf{I})^{\top}(\nabla \mathbf{u}+\mathbf{I})-\mathbf{I}\right)
$$

where $\mathbf{I}$ is the identity matrix, and $\nabla \mathbf{u}$ denotes the displacement gradient:

$$
\nabla \mathbf{u}:=\left(\begin{array}{ll}
\partial_{x_{1}} u_{1} & \partial_{x_{2}} u_{1} \\
\partial_{x_{1}} u_{2} & \partial_{x_{2}} u_{2}
\end{array}\right)
$$

In mechanical engineering, this tensor provides an important tool to derive properties of an observed material that undergoes a deformation. It describes how a motion differs locally from a rigid body motion. Later on, we are especially interested in the entry $e_{22}$. This is the strain in $x_{2}$-direction that describes how line elements parallel to the $x_{2}$-axis changed their length during the motion with respect to their initial length. A positive value indicates that a stretching has occured whereas a negative one indicates a compression. 
Let us now discuss if the optical flow field that minimises our energy functional (1) is suitable for computing this tensor. Here we are facing a problem: We remember that we are using the first order smoothness term (3) in our approach, which represents the idea that the entries of $\nabla \mathbf{u}$ should vanish. By considering the formula in (9), we find that this implies that the entries of the strain tensor should also vanish. So in practice, we can expect that using the derivatives of our solution $\mathbf{u}$ for computing $\mathcal{E}$ will lead to strain components that are biased towards zero.

This shows that the results of our baseline optical flow approach may not be suitable for deriving higher order motion information such as the strain tensor. Furthermore, Trobin et al. [미] observed that a first order smoothness term can lead to bad results for $\mathbf{u}$ itself if the true motion has a higher order smoothness.

Therefore, we conclude that we have to find variational approaches that, on the one hand, are able to directly estimate higher order motion derivatives, and that, on the other hand, use a higher order smoothness term in order to cope with complex motions.

\section{Extended Optical Flow Problem}

Let us now extend our baseline optical flow method in such a way that higher order smoothness is modelled and also motion derivatives are computed.

\subsection{Extended Variational Model}

As before, we consider the two frames $f_{1}$ and $f_{2}$ of our image sequence $f$. We now want to find a set $\Lambda_{n}$ of $n$ tensor-valued functions. It consists of the displacement field $\mathbf{u}=\left(u_{1}, u_{2}\right)$ and its derivatives up to order $n-1$. Let us illustrate this idea by investigating a few examples for $\Lambda_{n}$ : The case $n=1$ leads to $\Lambda_{1}=\{\mathbf{u}\}$, which is actually the classical optical problem. For $n=2$, we have $\Lambda_{2}=\{\mathbf{u}, \mathbf{A}\}$ where $\mathbf{A}=\left[a_{i j}\right]_{2 \times 2}: \Omega \rightarrow \mathbb{R}^{2 \times 2}$ contains the four first order derivatives of $\mathbf{u}$. By using $n=3$, we get $\Lambda_{3}=\{\mathbf{u}, \mathbf{A}, \mathbf{B}\}$ where the new $\mathbf{B}=\left[b_{i j k}\right]_{2 \times 2 \times 2}: \Omega \rightarrow$ $\mathbb{R}^{2 \times 2 \times 2}$ represents the eight second order derivatives of $\mathbf{u}$. As a convention, we will have in the following that $i, j, k \in\{1,2\}$.

Let us now consider suitable energy functionals for estimating $\Lambda_{n}$. Clearly, for $n=1$ we can just use our previous baseline approach:

$$
E_{1}(\mathbf{u})=\int_{\Omega}(D(\mathbf{u})+\alpha_{1} \underbrace{\sum_{i=1}^{2}\left\|\nabla u_{i}\right\|^{2}}_{=: S_{1}(\mathbf{u})}) \mathrm{d} \mathbf{x} .
$$

In order to model the problem for $n=2$, however, we also have to estimate $\mathbf{A}$, i.e. the first order derivatives of $\mathbf{u}$. This requires to modify our approach in (11). Instead of assuming that the first order derivatives of $\mathbf{u}$ vanish, we require that they should be similar to the entries of A, which in turn should have a first order smoothness. To this end, we replace the first order smoothness term $S_{1}(\mathbf{u})$ in (11) by a new expression that leads to the energy

$$
E_{2}(\mathbf{u}, \mathbf{A})=\int_{\Omega}\left(D(\mathbf{u})+\alpha_{1}(\underbrace{\sum_{i, j=1}^{2}\left(a_{i j}-\partial_{x_{j}} u_{i}\right)^{2}}_{=: M_{1}(\mathbf{u}, \mathbf{A})}+\alpha_{2} \underbrace{\sum_{i, j=1}^{2}\left\|\nabla a_{i j}\right\|^{2}}_{=: S_{2}(\mathbf{A})})\right) \mathrm{d} \mathbf{x}
$$


We see that A must fulfill the similarity term $M_{1}$, which causes it to contain estimates of the first order derivatives of $\mathbf{u}$. Variants of such a similarity term can also be found in the work of Corpetti et al. [ [ ] ]. Furthermore, we notice that the first order derivatives of $\mathbf{A}$ are expected to vanish, which is expressed by the smoothness assumption $S_{1}$. Finally, we see that $\mathbf{u}$ has to satisfy the data term $D(\mathbf{u})$ and the new term $M_{1}(\mathbf{u}, \mathbf{A})$. The latter term guarantees that the derivatives of $\mathbf{u}$ gain first order smoothness due to their similarity to the entries of $\mathbf{A}$. This causes $\mathbf{u}$ itself to have second order smoothness. Thus, we observe that our new expression solves two problems simultaneously: On the one hand, it allows us to directly estimate the first order derivatives of $\mathbf{u}$. On the other hand we have modelled a second order smoothness assumption for $\mathbf{u}$.

In order to make the recursive structure of this generic framework more explicit, let us now present the model for $n=3$ : Here we additionally have to find an estimate of $\mathbf{B}$ that contains the second order derivatives of $\mathbf{u}$. We can accomplish this by finding the estimates of the first order derivatives of $\mathbf{A}$. This motivates us to repeat the previous procedure: We replace $S_{2}(\mathbf{A})$ in (12) by a new expression. It consists of a similarity term between $\mathbf{B}$ and the derivatives of $\mathbf{A}$, and a first order smoothness term for $\mathbf{B}$. This gives

$$
\begin{gathered}
E_{3}(\mathbf{u}, \mathbf{A}, \mathbf{B})=\int_{\Omega}\left(D(\mathbf{u})+\alpha_{1}(\overbrace{\sum_{i, j=1}^{2}\left(a_{i j}-\partial_{x_{j}} u_{i}\right)^{2}}^{M_{1}(\mathbf{u}, \mathbf{A})}+\right. \\
\left.\alpha_{2}(\underbrace{\sum_{i, j, k=1}^{2}\left(b_{i j k}-\partial_{x_{k}} a_{i j}\right)^{2}}_{:=M_{2}(\mathbf{A}, \mathbf{B})}+\alpha_{3} \underbrace{\left.\sum_{i, j, k=1}^{2}\left\|\nabla b_{i j k}\right\|^{2}\right)}_{:=S_{3}(\mathbf{B})})\right) \mathrm{d} \mathbf{x} .
\end{gathered}
$$

Here the term $S_{3}(\mathbf{B})$ guarantees that $\mathbf{B}$ has first order smoothness. Moreover, $M_{2}(\mathbf{A}, \mathbf{B})$ causes $\mathbf{B}$ to contain estimates of the first order derivatives of $\mathbf{A}$. This implies that $\mathbf{A}$ will have second order smoothness. Finally, $M_{1}(\mathbf{u}, \mathbf{A})$ ensures third order smoothness for $\mathbf{u}$.

It is evident how one can continue this recursion to solve the extended optical flow problem for arbitrary orders $n$. Thus, we have established a generic framework for modelling higher order smoothness assumptions for the optical flow field, and for the stable estimation of higher order motion derivatives. This is exactly what we wanted.

\subsection{Euler-Langrange Equations and Boundary Conditions}

Since we have to minimise the energy functionals of our framework, let us now state the associated Euler-Lagrange equations and their corresponding boundary conditions. For the sake of brevity we restrict ourselves to the energy (13) that arises for $n=3$ : It contains all types of equations and conditions that appear for functionals with $n>1$.

We observe that $\mathbf{u}=\left(u_{1}, u_{2}\right)$ appears in the data term $D(\mathbf{u})$ and in the similarity term $M_{1}(\mathbf{u}, \mathbf{A})$. This leads to two Euler-Lagrange equations (one for $u_{1}$, one for $u_{2}$ ):

$$
0=\partial_{u_{i}} D(\mathbf{u})+\alpha_{1}\left(\partial_{x_{1}} a_{i 1}+\partial_{x_{2}} a_{i 2}-\Delta u_{i}\right) \text { for } i \in\{1,2\} \text {. }
$$

The component $\mathbf{A}$ is involved in the two similarity terms $M_{1}(\mathbf{u}, \mathbf{A})$ and $M_{2}(\mathbf{A}, \mathbf{B})$, which gives rise to four equations:

$$
0=a_{i j}-\partial_{x_{j}} u_{i}+\alpha\left(\partial_{x_{1}} b_{i j 1}+\partial_{x_{2}} b_{i j 2}-\Delta a_{i j}\right) \quad \text { for } i, j \in\{1,2\} .
$$


Next we turn to $\mathbf{B}$ : It participates in the similarity term $M_{2}(\mathbf{A}, \mathbf{B})$ and in the smoothness term $S_{3}(\mathbf{B})$. This creates eight Euler-Lagrange equations:

$$
0=b_{i j k}-\partial_{x_{k}} a_{i j}-\alpha \Delta b_{i j k} \quad \text { for } i, j, k \in\{1,2\} .
$$

Finally we treat the boundary conditions. Here we can identify two different types:

$$
\begin{aligned}
\mathbf{n}^{\top} \nabla b_{i j k} & =0 \quad \text { for } i, j, k \in\{1,2\}, \\
\mathbf{n}^{\top}\left(\begin{array}{l}
b_{i j 1}-\partial_{x_{1}} a_{i j} \\
b_{i j 2}-\partial_{x_{2}} a_{i j}
\end{array}\right) & =0 \quad \text { for } i, j \in\{1,2\}, \\
\mathbf{n}^{\top}\left(\begin{array}{l}
a_{i 1}-\partial_{x_{1}} u_{i} \\
a_{i 2}-\partial_{x_{2}} u_{i}
\end{array}\right) & =0 \quad \text { for } i \in\{1,2\} .
\end{aligned}
$$

Equation (17) represents the first type of boundary conditions. It originates from the first order smoothness term $S_{3}(\mathbf{B})$. The second type appears in (18) and (19). It emerges from the similarity terms $M_{1}(\mathbf{u}, \mathbf{A})$ and $M_{2}(\mathbf{A}, \mathbf{B})$, respectively.

\section{Discretisation and Implementation}

Since our image data are digital, we have to find discrete versions of our Euler-Lagrange equations and their boundary conditions. To this end we apply classical finite difference methods; see e.g. [ $\square$ ] for an introduction to this field of numerical analysis. Since our data term (2) is a nonquadratic function of the flow field $\mathbf{u}$, this discretisation process leads to a nonlinear system of equations. We solve it iteratively with a variant of the Gauss-Seidel algorithm in which we have frozen the nonlinear coefficients; see also [ $\mathbf{Q}, \mathbf{\square}, \mathbf{Q}]$. Furthermore, we perform an incremental computation of the displacement parts $u_{1}$ and $u_{2}$ in order to handle large motions in a better way. This is known as warping and requires the linearisation of the data term and embedding the solving process into a multiresolution framework [ $\square]$ ]. For the experiment below, our unoptimised ANSI C implementation on a single core of an Intel Core i7-3770 CPU requires about 1 minute for $n=1,30$ minutes for $n=2,100$ minutes for $n=3$, and 210 minutes for $n=4$ to analyse an image pair with $225 \times 246$ pixels. We expect that substantial speed-ups can be achieved with parallel algorithms for GPU implemetations, if this becomes necessary.

\section{Experimental Evaluation}

To evaluate our generic variational framework for the extraction of motion derivatives such as the Lagrangian strain tensor, we first have to design a suitable experiment. It should involve a motion that is sufficiently complex to justify the usage of higher order smoothness terms and where the strain tensor is of interest.

Our experimental setting is shown in Figure 1. It depicts two frames of a biaxial tensile experiment with an elastomer. The material sample is stretched along the $x_{1}$ - and $x_{2}$-axis. Such experiments provide useful tools in mechanical engineering to accurately inspect the behaviour of a material [ㅁ] . Furthermore, Promma et al. [ㅁ] ] have found that the displacement gradient $\nabla \mathbf{u}$ associated with such a motion shows a non-constant behaviour in space, which implies that the motion is sufficiently complex for our needs. We use the two areas 

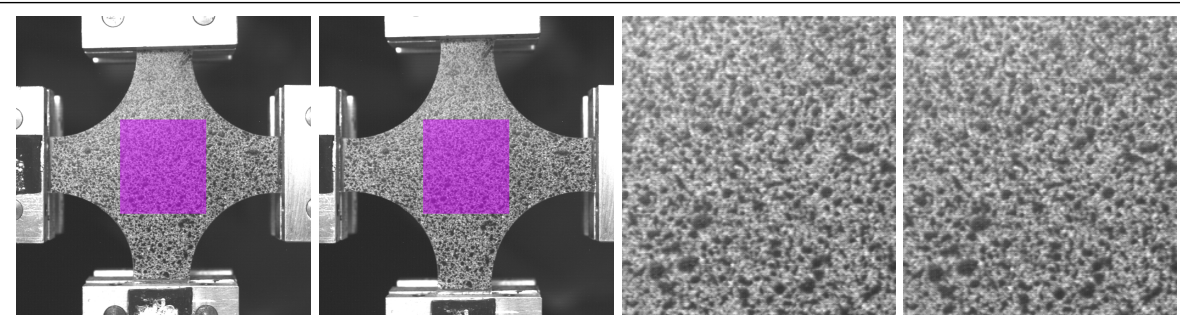

Figure 1: (a) Left: Two frames of the biaxial tensile experiment with the areas of interest colored in purple. (b) Right: Enlarged versions of the respective areas of interest.

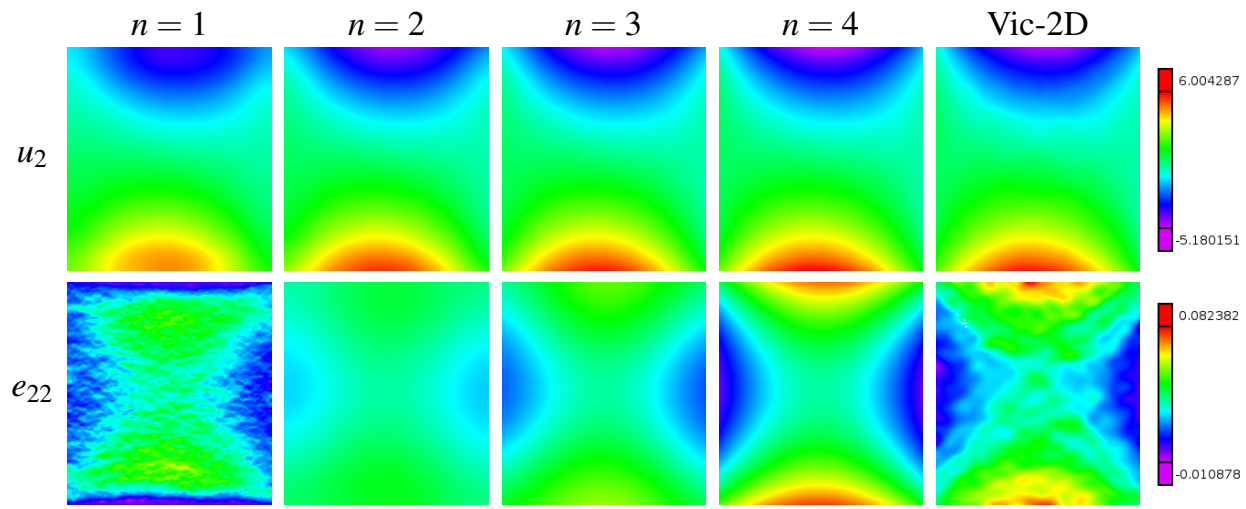

Figure 2: Comparison between our variational approach with different orders $n$ (using $\alpha_{i}=$ 2000) and the commercial software Vic-2D. The top row shows the optical flow component $u_{2}$, and the bottom row depicts the Lagrangian strain tensor component $e_{22}$. For $n=1$, the result for $e_{22}$ has been obtained by numerical differentiation of $u_{2}$.

of interest in Figure 1 as our images $f_{1}$ and $f_{2}$ and solve the extended optical flow problem. Since these pictures are taken from a real-world experiment, no ground truth is available. In order to assess the correctness of our results, we use the results of the commercial software Vic-2D as a reference solution. This specialised software belongs to the leading tools for evaluating data of such experiments. It is based on sophisticated digital image correlation techniques [四].

The results of our experiment are visualised in Figure 2 where we analyse both the optical flow component $u_{2}$ in $x_{2}$ direction and the Lagrangian strain tensor component $e_{22}$ in $x_{2}$ direction. As model orders $n$ we have used values between 1 and 4 . Interestingly all approaches give visually very similar results for the optical flow component $u_{2}$, while differing substantially in the outcome for the Lagrangian strain tensor component $e_{22}$. This shows that the reliable estimation of motion derivatives is substantially more difficult than a classical optical flow problem, confirming one more time the ill-posedness of differentiation. In particular, we also see that a visually smooth flow field does by no means guarantee a qualitatively useful Lagrangian strain tensor field.

Thus, let us now have a closer look at the results for the strain tensor component $e_{22}$. For $n=1$, we have computed it via a numerical differentiation of the optical flow by means of 
central differences of second order. Thus, this result shows the naive approach that combines a variational optical flow method with a postprocessing by differentiation. As expected the outcome is very noisy and differs a lot from the reference solution of Vic-2D.

For $n \geq 2$, we obtain results that are directly computed within our novel variational framework. Using the order $n=2$ gives a very flat strain value that seems to be almost constant. This can be explained as the effect of $n=2$ that leads to a variational approach with a second order smoothness term. It encourages $\nabla \mathbf{u}$ to be constant in space. The substantial differences to Vic-2D shows that we rather observe artifacts of our model than real physical phenomena. Thus, our result for $e_{22}$ can not be regarded as correct for $n=2$.

With higher orders for $n$ the result comes closer to the reference solution. Interestingly even $n=3$ is not sufficient to give a satisfactory strain estimate, which may be explained by the relatively complex motion structure in our experiment. However, increasing $n$ to 4 finally yields a strain tensor field that shows the desired non-constant behaviour in space and has a similar structure as the reference solution. Furthermore, the symmetry of the result for $e_{22}$ corresponds to the observations made by Chen et al. [0]. Thus, we conclude that this time our result can be regarded as correct. Moreover, it even ourperforms the outcome of Vic-2D in terms of its impressive robustness under noise.

\section{Conclusions}

In our paper we have introduced a generic variational framework for the stable estimation of motion derivatives in image sequences. Due to its recursive model structure, it can be extended to arbitrary high orders in a straightforward way. As an example application we have focused on the computation of the Lagrangian strain tensor which is an important problem in mechanical engineering. Here we have shown that our framework can give state-of-the-art results.

For computer vision researchers it may appear surprising that fourth order variational models are needed to give the desired results: Most models that are used so far are of first order and only a few of second order. Our research shows that it is both computationally feasable and practically rewarding to go to higher orders. While we have focused on quadratic smoothness terms, it is no problem to replace them by their nonquadratic or even anisotropic counterparts. This is studied in our ongoing research.

For mechanical engineers our results have demonstrated that computer vision ideas can substantially improve measurement technology. Moreover, a stable evaluation of even higher order derivatives than the ones that are covered by classical theories is no longer an illusion. This may give novel insights in the behaviour of materials and even fertilise new theories.

\section{References}

[1] M. A. Sutton, J. Orteu, and H. Schreier. Image Correlation for Shape, Motion and Deformation Measurements: Basic Concepts, Theory and Applications. Springer, New York, 2009. 
[2] L. Alvarez, C. A. Castaño-Moraga, M. García, K. Krissian, L. Mazorra, A. Salgado, and J. Sánchez. Second order variational optic flow estimation. In Proc. 2007 International Conference on Computer Aided Systems Theory, pages 646-653, 2007.

[3] M. Bertero, T. A. Poggio, and V. Torre. Ill-posed problems in early vision. Proceedings of the IEEE, 76(8):869-889, August 1988.

[4] K. Bredies, K. Kunisch, and T. Pock. Total generalized variation. SIAM Journal on Imaging Sciences, 3(3):492-526, 2010.

[5] A. Bruhn and J. Weickert. Towards ultimate motion estimation: Combining highest accuracy with real-time performance. In Proc. Tenth International Conference on Computer Vision, volume 1, pages 749-755, Beijing, China, October 2005. IEEE Computer Society Press.

[6] Z. Chen, T. Scheffer, H. Seibert, and S. Diebels. Microindentation of a soft polymer: Identification of hyperelasticity and validation by uni/biaxial tensile tests. Mechanics of Materials, 2013. Submitted.

[7] T. Corpetti, E. Mémin, and P. Pérez. Dense estimation of fluid flows. IEEE Transactions on Pattern Analysis and Machine Intelligence, 24(3):365-380, March 2002.

[8] C. Frohn-Schauf, S. Henn, and K. Witsch. Nonlinear multigrid methods for total variation image denoising. Computing and Visualization in Science, 7(3-4):199-206, 2004.

[9] I. M. Gelfand and S. V. Fomin. Calculus of Variations. Dover, New York, 2000.

[10] B. Horn and B. Schunck. Determining optical flow. Artificial Intelligence, 17:185-203, 1981.

[11] K. W. Morton and L. M. Mayers. Numerical Solution of Partial Differential Equations. Cambridge University Press, Cambridge, UK, second edition, 2005.

[12] R. W. Ogden. Non-linear Elastic Deformations. Dover, New York, 1997.

[13] N. Papenberg, A. Bruhn, T. Brox, S. Didas, and J. Weickert. Highly accurate optic flow computation with theoretically justified warping. International Journal of Computer Vision, 67(2):141-158, April 2006.

[14] N. Promma, B. Raka, M. Grédiac, E. Toussaint, J. B. Le Cam, X. Balandraud, and F. Hild. Application of the virtual fields method to mechanical characterization of elastomeric materials. International Journal of Solids and Structures, 46(3):698-715, 2009.

[15] L. R. G. Treloar. The Physics of Rubber Elasticity. Oxford University Press, Oxford, 2005.

[16] W. Trobin, T. Pock, D. Cremers, and H. Bischof. An unbiased second-order prior for high-accuracy motion estimation. In G. Rigoll, editor, Pattern Recognition, volume 5096 of Lecture Notes in Computer Science, pages 396-405. Springer, 2008. 